\title{
Diversity in seed longevity amongst biodiverse seeds
}

cambridge.org/ssr

\section{Commentary}

Cite this article: Pritchard HW (2020). Diversity in seed longevity amongst biodiverse seeds. Seed Science Research 30, 75-80. https://doi.org/10.1017/S0960258520000306

First published online: 2 September 2020

Author for correspondence:

Hugh W. Pritchard,

h.pritchard@kew.org

\section{Hugh W. Pritchard}

Royal Botanic Gardens, Kew, Wakehurst Place, Ardingly, West Sussex RH17 6TN, UK

\section{Introduction}

The degradation of nature is among the most serious issues that the world faces, with pressing needs to simultaneously avoid dangerous climate change, feed a growing population and restore biodiversity (Mace et al., 2018). Within a balanced, wide-ranging and cross-cutting response to counteract all three challenges, there is an accepted role for plant diversity, including planting trees (climate emergency), the development of stress-tolerance crops (food security) and landscape recovery (restoration of biodiversity). All such actions depend on an adequate supply of high-quality seeds at the right time and place. This makes understanding the factors that control seed longevity, whether in the in situ or ex situ environment, fundamentally important to successful outcomes. Progresses in the understanding of this topic presented at the recent ISSS Seed Longevity Workshop in Fort Collins, USA, are synthesized here. What is evident is that seed longevity continues to span fundamental and applied science, is increasingly multidisciplinary, and is advancing rapidly with the application of a greater range of molecular and biophysical tools to dissect responses under a wide range of environmental conditions.

\section{Banking for the future}

Understanding seed longevity is of exceptional importance for germplasm conservation, as the vast majority of the 7.4 million gene bank accessions of plant species stored globally are of seeds, stored dry and cold. Examples include about 150,000 accessions at IPK, Germany (Andreas Börner, Germany), about 130,000 accessions at the International Rice Research Institute (Jae-Sung Lee, Philippines), 3000 accessions of PGR held at the Bank of Plant Genetic Resources of the Agricultural University of Georgia (Mariam Betsiashvili, Georgia) and $>35,000$ species' seeds duplicated in the Millennium Seed Bank of Kew (John Dickie, UK). Because of the International Treaty for PGR for Food and Agriculture, seed material can be exchanged freely between the crop banks. Thus, the genebank at the AU Georgia holds unique Georgian PGR from foreign genebanks (IPK, USDA and CIMMYT). In contrast, wild species banks transfer material under Convention on Biological Diversity-compliant access and benefit-sharing agreements. Exchanging and distributing banked accessions are extremely important activities in light of the current wide range of societal challenges associated with sustainable development, the biodiversity crisis and the climate emergency. In this context, banking and exchanging pollen is also a valuable endeavour, particularly to support breeding programmes for climate-resilient crops. But the challenges can be considerable. For hybrid wheat, fresh pollen vitality can be rapidly lost (minutes) at room temperature, while $6-8^{\circ} \mathrm{C}$ extends survival and increases germination, indicating the presence of a dormancy mechanism (Manuela Nagel, Germany).

As banking technology and the main purpose of a bank can change over time, process and storage conditions might be altered. At the Alberta Tree Improvement \& Seed Centre tree seed bank, the initial storage temperature was $2^{\circ} \mathrm{C}$, with the samples moved to $-18^{\circ} \mathrm{C}$ storage in the mid-1980s. Greater consideration is now given to genetic diversity and the need to meet new government regulations on minimum viability standards for seed usage, to the longer-term benefit of researchers and industry (Lindsay Robb, Canada). There are also new seed bank initiatives, such as Missouri Botanical Garden, which conserved nearly 4000 accessions of 761 taxa by 2018 (Meg Engelhardt, USA). It aims to integrate the conservation of horticultural and research collections and to seed bank Missouri's entire native flora, with a strong focus on species conservation status (e.g. conservation concern and globally rare), eco-region fragility, redundancy in other ex situ collections, and crop wild relative status.

An essential part of all seed bank operations is the high standard of curation of accessions of known provenance (from a wide range of countries and sites) and storage history. Indeed, there are now historical germination data accumulated over many decades of viability monitoring during storage (Andreas Börner, Germany; John Dickie, UK). These accessions are being used to refine questions about inter- and intra-specific differences in longevity, using genetic analyses, metabolic profiling (e.g. lipid oxidation), phylogenetic and biogeographical 
perspectives, and to conduct longitudinal studies in relation to changing climate. However, the subsequent widespread use of this technology has meant that 'the diversity of seeds to curate is an ever-increasing challenge for managers of seed banks' (David Merritt, Australia). This includes all four stages of the process: harvesting, drying, storing and germinating. Indeed, the seed banking and use of globally threatened plants can be constrained by small numbers of potentially viable or usable seeds (Liu et al., 2020).

\section{Drying might mean dying}

Generally, seeds stored for conservation are pre-dried in equilibration with around $15-25 \% \mathrm{RH}$ and within the seed trade often to around $35-40 \% \mathrm{RH}$. But not all species produce seeds that tolerate such treatment, including potentially orthodox seed when harvested prematurely. In limber pine (Pinus flexilis, Pinaceae), seed harvest timing is difficult to perfect. Visual cues in the field can be unreliable, as some seed with embryos occupying $>90 \%$ of the cavity are not yet desiccation-tolerant or have acquired maximum longevity. While ex situ ripening of cones (1-6 weeks) increases seed germination and desiccation tolerance, the seeds are very short-lived; making their storage and use in restoration difficult (Lindsay Robb, Canada). In contrast, mature seeds of Alnus glutinosa, Betula pendula, Carpinus betulus (all Betulaceae) and Prunus avium (Rosaceae) can be desiccated to about 2\% MC and stored conventionally at low temperature with relative impunity; while Fraxinus excelsior (Oleaceae), Malus sylvestris (Rosaceae), Tilia cordata (Malvaceae), Salix spp., Populus spp. (both Salicaceae) and Fagus sylvatica (Fagaceae) seeds have a slight sensitivity to drying below $5-7 \%$ MC (Paweł Chmielarz, Poland). Campomanesia adamantium (Myrtaceae), which has phytotherapeutic potential and fruits with high vitamin $\mathrm{C}$, produces a seed that survives drying to $15 \%$ but not 5\% MC (Érica Fernandes Leão-Araújo, Brazil). Much more intolerant of drying are species in the oak and chestnut genera Quercus and Castanea (both Fagaceae), being sensitive below about 40\% MC (Paweł Chmielarz, Poland; Hongying Chen, China). In the case of Castanea mollissima, molecular dissection of the response using lipidomics shows that the translocation of phospholipase D $\alpha 1$ from the cytosol to the membrane is more prevalent in sensitive seeds and the accumulation of high levels of phosphatidic acid in the membrane correlates with viability loss (Hongying Chen, China). Because of the high prevalence of seed desiccation sensitivity in threatened trees, achieving global ex situ conservation targets through conventional seed banking alone is predicted to be unachievable, according to a model based on boosted regression trees (John Dickie, UK). This means that all means of preserving threatened trees should be explored, including pollen banking. Interestingly, desiccationtolerant pollen is quite common among tree species of temperate origin and can be independent of the seed's level of desiccation tolerance; for example, Corylus vellane, Corylus colurna (Betulaceae), Taxus baccata (Taxaceae) and Ilex aquifolium (Aquifoliaceae) have more tolerant seeds, and Quercus species and Aesculus (Sapindaceae) species have less tolerant seeds (Dani Ballesteros, UK). But sensitivity to drying is not restricted to tree seeds, and the longevity of recalcitrant seeds of Northern Wild Rice (Zizania palustris, Poaceae) can be extended by avoiding drying and storage fully hydrated at close to $0^{\circ} \mathrm{C}$ (Jacques Duquette, USA).

\section{Predicting outcomes}

Assuming the seed survives drying it is of the utmost interest to understand and even predict subsequent longevity, as this is a key consideration in germplasm management. Traditional approaches to monitoring the viability of stored seeds are labour intensive and sometimes 'reactionary', offering indications of when re-collection is essential, but with limited predictive capacity (Emma Dalziell, Australia). Nonetheless, germination testing remains a routine part of collections management, with regular viability monitoring for almost 17,000 collections in the Millennium Seed Bank being used for decision support in (re)collection targeting and management (John Dickie, UK). Analysing historical, monitoring data is equally important for the CGIAR genebanks, for example among 35 crop and tree collections, some of which have been held for more than 50 years (Ramad Jawad, Lebanon). When the majority of accessions are stored at $4^{\circ} \mathrm{C}$, as at the North Central Plant Introduction Station (NCRPIS) in Ames, Iowa, regular monitoring becomes even more important, particularly as there is evidence for a 'germination cliff in maize accessions, with the risk of impacting the genetic profile of an accession and/or preservation of rare alleles (Lisa A. Burke, USA). Monitoring of seed viability of Hawaiian lobeliads stored dry $(\sim 20 \% \mathrm{eRH})$ and cool $\left(5^{\circ} \mathrm{C}\right)$ for up to 24 years showed nine species to have short lifespans, with P50s between 1.4 and 8.5 years, based on three different survival curve fitting methods. Hawaii is a biodiversity hotspot for speciation in lobeliads (Campanulaceae) with 159 taxa, all of which are endemic, making the analysis of survival curves critical to decision-making regarding the security of rare plant conservation through seed banking and time intervals to regeneration and/or, where possible, recollecting (Dustin Wolkis, USA). Such decision-making also needs to consider operational value for money, as the maintenance of PGR generate specific costs over long time intervals, particularly cold $\left(-18^{\circ} \mathrm{C}\right)$ versus ultra-low temperatures $\left(-196^{\circ} \mathrm{C}\right)$. Based on comparisons for a range of (mainly) crops, a spreadsheet is now applied to find the breakeven point and rational explanation for a specific bio-banking scenario. Accounting for pollination and breeding type, multiplication cycle, seed longevity, required working time, equipment and consumables, etc., shows that for the best outcome a single set of storage conditions may not be appropriate for all species (Manuela Nagel, Germany).

Although species characteristics for seed longevity can provide estimates of seed longevity in storage (Yong-bi Fu, Canada), and some patterns in longevity are noticeable across the plant kingdom (Colville and Pritchard, 2019), much remains to be discovered about seed longevity in biodiverse species. There are some correlations between physiological and molecular features of forest tree seeds and seedlings after seed storage, which can be useful for the early determination of seed ageing during storage in (forest) gene banks in the future (Pawel Chmielarz, Poland). And there is much evidence for an association between seed persistence and seed mass, with smaller seeds tending to be more likely to produce persistent in situ seed banks and to have good longevity. Interestingly, recent evidence suggests a role for intra-specific variation in seed mass in ex situ storage conditions, not just in differential germination strategies. When Rudbeckia mollis (Asteraceae) seeds of three mass classes are subjected to saturated salt accelerated ageing (SSAA; $\left.41^{\circ} \mathrm{C}, 75 \% \mathrm{RH}\right)$, the two lighter classes are more than twice as likely to germinate after 6 months storage compared to the heavier seeds (Nicholas Genna, USA). Moreover, under controlled deterioration, bigger seeds of 
dimorphic Aegilops (Poaceae) tend to be shorter-lived and germinate faster than smaller seeds in most of the species studied. General antioxidant activity is significantly higher in the smaller seeds of most of the species in a comparison of 18 different seed morphs amongst six Aegilops and Triticum (Poaceae) species, perhaps also reflecting different soil seed bank persistence (Filippo Guzzon, Italy). These findings suggest that accelerated ageing should be used with predictive caution and that seed mass and longevity should be studied within accessions (Nicholas Genna, USA). Hopefully, such studies can be advanced within a wider range of species in the $5 \%$ of angiosperm families in which diaspore heteromorphism occurs.

While evidence emerges of some predictability of outcomes based on seed mass, phylogeny, biogeography, etc., considerable interest, and controversy, remains about the extrapolation of longevity performance under one set of environmental conditions to another; and indeed, how much the mechanisms of seed viability loss vary with storage conditions. For example, rice ageing under quick CD/AA tests often shows poor correlation with long-term storage under dry conditions (C.T. Manjunath Prasad, Netherlands). Pinus densiflora (Pinaceae) seed $\alpha$-tocochromanol shows significant differences only under seed bank $\left(-20^{\circ} \mathrm{C}, 40 \%\right.$ $\mathrm{RH})$ ageing (SA), while seed glutathione contents and GSH halfcell reduction potential is distinctly changed only under accelerated ageing ( $\left.\mathrm{AA} ; 45^{\circ} \mathrm{C}, 80 \% \mathrm{RH}\right)$; and volatiles $\beta$-pinene and $\alpha$-pinene decrease in AA seeds but not obviously in SA seeds. So the biochemical characteristics for AA or SA seeds are not exactly the same (Chae Sun $\mathrm{Na}$, South Korea). Moreover, various SSAA results do not reliably predict differences in deterioration rates following laboratory storage for Rudbeckia hirta var. angustifolia (Asteraceae) seeds (Tia Tyler, USA). When seeds of winter wheat genotypes stored for up to 16 years at either ambient $\left(20^{\circ} \mathrm{C}\right.$; losing viability) or cold storage $\left(0^{\circ} \mathrm{C}\right.$; high viability) are multiplied and fresh seeds subjected to controlled seed deterioration at two MCs, all show significant changes in reduced and oxidized glutathione. However, changes in $\mathrm{pH}$ are only observed in the dry (lower MC) aged seeds, suggesting that the physical state of the seeds during storage may affect the biochemical processes that lead to seed deterioration in different ways (Manuela Nagel, Germany).

When comparisons are being made between storage times that span days (for accelerated conditions at high $\mathrm{RH}$ ) to years (lab and bank storage at low RH), it seems that the relative importance of specific pathways modulating the ageing process will not be exactly the same. Thus, the choice of seed ageing environment might change the findings of genetic and other types of studies on seed longevity/storability (Hay et al., 2019).

\section{Genes}

Clearly, more science-based methodologies for selecting seeds used for regeneration, and monitoring seed ageing around molecular biology, genomics and seed ageing signals, is needed in order for germplasm collection curators to preserve the initial genetic profiles of accessions (Lisa A. Burke, USA; Yong-bi Fu, Canada). Dry seeds exist in the vulnerable state of being unable to repair their chemical degradation which slowly ages seeds and eventually results in death. Proposed seed ageing mechanisms involve all classes of biological molecules (Margaret Fleming, USA), meaning that there is wide scope for the development of molecular tools. Because the conditions used for seed ageing studies are often conducive to changes in seed lot dormancy status, taking a neural network approach might be particularly valuable in helping to disentangle signalling pathways and to unravel cause-effect relationships between the time of death during storage and deteriorative reactions leading to seed ageing (Zinsmeister et al., 2020).

Based on an inference of expressed genes approach, transcription factor PROGERIA1 (PRO1) - a member of the APETALA2/ EREBP family - may be involved in seed longevity in Medicago truncatula (Leguminosae). Seeds from mutants deficient in this TF have much-reduced longevity, combined with severely impaired regulation of photosynthesis and chloroplastic genes, and much higher chlorophyll in dry seeds cf. WT. It could also act in concert with the ABA signalling pathway between desiccation tolerance and longevity (Olivier Leprince, France). Degradation of the transcriptome (total mRNA) occurs contemporaneously with viability loss in long-term (years) dry-stored seeds of the soybean (Glycine max, Leguminosae). Non-specific fragmentation in longer transcripts is consistent with the proposed mechanism of molecular fission by free radical attack at random bases, and high integrity of short transcripts are not sufficient to maintain viability (Margaret B. Fleming, USA). DNA methylation (MSAP) and degradation (RAPD) may also be a feature of rapid (warm, humid conditions) seed ageing, with epigenetic changes impacting gene expression in Mentha (Lamiaceae) seeds in response to ageing. In addition, the induction of secondary dormancy during storage complicates viability assessment (Sara Mira, Spain). Use of mitochondrial DNA (mtDNA) specific primers shows impaired energy metabolism, and changes in ATP synthase and $\mathrm{NADH}$ genes in wheat seeds during ageing in various containers and relatively high MC (14\%). In contrast, genes of $\mathrm{NADH}, \mathrm{ATP}$ synthase and cytochrome $\mathrm{b}$ and $\mathrm{c}$ type are found to remain intact in seeds in hermetic storage at relatively low MC (8\%) (I. Afzal, Pakistan).

In Arabidopsis thaliana (Brassicaceae), QTLs for germination ability after storage (GAAS) are evident when using EPPO (Elevated Partial Pressure of Oxygen) ageing but not from artificial ageing (i.e. increased humidity and temperature). For the first phase of the seed lifespan (i.e. dormancy release), DOG loci are revealed (Gonda Buijs and Leónie Bentsink, Netherlands). Similarly, in rice, specific and shared genetic bases controlling seed storability and dormancy have been found. In a weed/cultivar cross in Oryza, 10 QTLs are associated with seed storability at $25^{\circ} \mathrm{C}$ and four of these co-locate with known genes/QTL for seed dormancy (e.g. ABA, flavonoid, GA and seed shattering). The remaining storability loci include a major effect QTL ( $>50 \%$ population variance) influencing seed viability through the embryo tissue. Thus, two sets of genes underlying the genotype variation of seed longevity in storage relate to a major set evolved specifically for storability and the other set shared with or influenced by seed dormancy (Xing-You Gu, USA).

Metabolomics is also revealing the complexity of the seed longevity trait. In two-hybrid cultivars of rice, two- to six-times as many metabolites and proteins change during natural ageing in the shorter-lived cultivar (IIT998) compared with the longer-lived one (BY998). This includes glutathione-related proteins, and levels of most sugar-related metabolites are significantly higher in IIY998 seeds both before and after storage (J. Liu, China). In genotypes of rice with allelic variation in the Rc gene - specifically a bHLH TF that regulates pro-anthocyanidin (PAs) synthesis there is variation in seed tolerance to dry EPPO ageing (C.T. Manjunath Prasad, Netherlands). This ties in with the importance of oxidative stress in seed longevity (viability loss) as PAs are an 
important subclass of flavonoids and have strong antioxidant activity. There is an important role for vitamin $\mathrm{E}$ (i.e. $\alpha-, \beta-, \gamma^{-}$ and $\delta$ - tocopherol/tocotrienol) in varying seed longevity among 200 'Aus' accessions of rice based on GWA mapping (using $\mathrm{SNP}$ ); their unique chromanol ring structure effectively scavenges lipid peroxy radicals which cause membrane breakdown during seed ageing (Jae-Sung Lee, Philippines). In rye (Secale cereale, Poaceae) cv. Dańkowskie Złote seeds stored dry (about 5\% MC) for 40 years at a range of temperatures $\left(20\right.$ to $\left.-18^{\circ} \mathrm{C}\right)$, air storage is less beneficial than storage in inert atmospheres. Volatile aldehyde emission during imbibition correlates with seed viability, suggesting the initiation of repair to unsaturated lipids probably in cell membranes (Andreas Börner, Germany).

\section{Molecular mobility}

Specific molecular networks clearly prepare the seed for entering into the dry state and contribute to the recovery of functioning cells during imbibition after storage. However, drying the cytoplasm induces solidification and inhibition of metabolic reactions. Once the solid phase is formed, the question of survival changes from if to when. The 'when' is regulated by seed quality factors as well as storage conditions. Ageing is an accumulation of random damage to molecules of diverse functions and importance to survival, with mortality a large and unpredictable response to the cumulative small reactions that cannot be stopped even at cryogenic temperatures (Christina Walters, USA). While solidification generally prolongs lifespan, longevity still varies within and among species, and this seems to depend on complex physicalchemical interactions within this 'dry architecture' involving chloroplasts and storage lipids and their predilection for crystallization (D. Ballesteros, UK). Lipid crystallization may be a feature of relatively poor seed longevity in many species of the Hawaiian flora. Among 400 taxa stored at both 5 and $-18^{\circ} \mathrm{C}$, significant freeze-sensitivity is seen in Campanulaceae, Cyperaceae, Rubiaceae, Santalaceae and Urticaceae, resulting in greatly varying estimates for re-collection intervals (based on 70\% of highest germination recorded) from $20+$ to $<5$ years (Marian M. Chau, USA). Interestingly, seeds from bitter gourd (Momordica charantia, Cucurbitaceae) apparently have freeze-sensitivity as germination often fails after storage at sub-zero temperatures. Not only do those seeds retain viable embryos (tetrazolium staining), but heat $\left(45^{\circ} \mathrm{C}\right)$ application restores germination after storage for as long as 13 years at $-20^{\circ} \mathrm{C}$ (Steven P.C. Groot). As the response was highly reversible with multiple cold treatments, there are implications for collections management of oil-rich seeds in the future.

\section{Post-storage use}

Seed is capital banked and, as with other forms of capital, it should be made available for use. Probably, one of the best examples of this is the USDA National Plant Germplasm System (NPGS). Its network of genebanks annually distributes 250,000 accessions worldwide for use in research and plant breeding programmes (Stephanie Greene, USA). To facilitate distribution, it is often the case that seed accessions of annual crops are regenerated to ensure sufficient stock. In botanic gardens, the comparable situation involves the development and use of propagation protocols for plants from the living collection, as at Missouri Botanic Gardens (Meg Engelhardt, USA). Similarly, without the development of appropriate methods, seed dormancy will remain a major limitation in the commercial development of many native species. Some familiar interventions include the application of $\mathrm{H}_{2} \mathrm{SO}_{4}$ for $60 \mathrm{~min}$ to remove physical dormancy in Sesbania sesban (Fabaceae), a multi-purpose species (Ludy Keino, Kenya), and exposure of Plains bristlegrass (Setaria leucopila, Poaceae) seed to warm $\left(40^{\circ} \mathrm{C}\right)$ dry afterripening (John Reilley, USA). But for some species, considerable research investment to understanding the species ecology is needed, in a collaborative, multidisciplinary and multi-agency way, so that guidelines can be established for seed use in restoration. Such use is actively promoted by the International Network for Seed-based Restoration (INSR), for whom the main aim is to 'foster collaboration to develop standards for native plant seed testing and regulation'. By gathering and synthesizing published data and making it available to seed collectors, growers, practitioners and land managers, the supply and use of appropriate seed sources should increase. A good example is the publication of species profiles for North American native forbs: http://greatbasinfirescience.org/westernforbs-restoration/ (Nancy Shaw, USA).

The global commitments to restoring degraded landscapes in the next decade are considerable. In support of the Sustainable Developments Goals to conserve, restore and sustainably use terrestrial ecosystems (particularly forests, wetlands, mountains and drylands), the Bonn Challenge (https://www.bonnchallenge.org/content/challenge) aims 'to bring 350 million hectares of the world's deforested and degraded land into restoration by 2030'. Such action also aligns with other international conventions (e.g. the CBD Aichi Target 15, the UNFCCC REDD+ goal and the Rio+20 land degradation neutrality goal). With such a burgeoning of policy, restoration is (about to become) big business, and most restoration initiatives depend on quality seeds. One concern is that the majority of seed used will be from easily available commercial species (e.g. trees and fodder species), and rare plant reintroduction will suffer. Thus, the increased efficacy of using seeds to establish rare populations is important. The endangered legume Dalea carthagenensis var. floridana has hard seeds, and the seeds remain viable for $1452 \mathrm{~d}$ even when moist. Only seed introduced to natural habitat, rather than novel habitat, grow into plants that mature, reproduce, and establish seedlings. This is a good example of seed augmentation helping to rescue an endangered population from the brink of extinction (Joyce Maschinski, USA). Clearly, an ecologically guided approach to identifying seed and plant traits that support seed survival and regeneration contributes to the effective use of seeds in returning native plant communities to degraded habitats (David Merritt, Australia). Such insight helps decisions on when, where and what to seed. For example, using an understanding of the timing of germination (impacted by dormancy) for the successful regeneration of forbs across 17 locations within the Great Basin sagebrush steppe (SC Barga, Univ Nevada, USA). Also, life-history strategies are important for the persistence of rare Australian alpine daisies species. Common daisies of the Brachyscome (Asteraceae) genus exhibit higher germination across most habitats (heathland $v$ s tussock or rocky grasslands), but rare species maintain a higher proportion of viable seed in all seedbed conditions (Megan J. Hirst, Australia). Also, the spatial and temporal variability in seedbed microclimate can facilitate an assessment of within- and between-population variability in genotype and phenotypic responses. Having a defined metric for a trait that is spatially structured lends itself to a 'reverse ecology' approach to partitioning genetic variation (polymorphism). This is a cost- 
effective way of generating data for use in multiple populations and locations to address population variability in stand establishment, including seed emergence (persistence in soil seed bank), germination rate and natural seed priming processes in the in situ soil seed bank (Christopher M. Richards, USA).

\section{Innovative technologies}

The longevity of seed in storage is influenced by storage conditions and the initial quality of the seed, and gentle handling is known to be of importance to longevity (Solberg et al., 2020). Of course, there are many different aspects of seed quality of interest to the seed trade and farmers as they describe the condition and value of their seed lots. Because more traditional seed testing methods are time-consuming and require highly skilled seed analysts, techniques that promise faster, efficient and nondestructive seed testing are under development and are in great demand by the seed sector. These include machine vision, Raman and IR spectroscopy, hyper-spectral and thermal imaging, $\mathrm{X}$-rays and chlorophyll fluorescence to assess a range of quality indicators, such as chemical composition, purity, disease, mechanical damage, insect damage, seed vigour and viability (Laura Bowden, Scotland). More traditional methods of determining seed quality remain important for many species, such as seed weight, seed width, seed thickness and seed coat colour among accessions of Bambara groundnut; seed weight showed a positive relation with germination percentage in 50 accessions (Temitope Mary Jones, Nigeria). Moreover, small seed size and the associated dispersal structures in the leafy vegetable Crassocephalum crepidioides (Asteraceae) make post-harvest handling and preservation of the seeds difficult. In this species, longevity was negatively affected by physical damage to the seeds, indicating the need to combine careful post-harvest handling and modification of the storage environment (S.A. Ajayi, Nigeria). Not just seed size but the uniformity of shape is beneficial for efficiencies in storage and follow-up processing. For example, polishing (to remove the soft outer pericarp) of sugar beet (Beta vulgaris; Amaranthaceae) and washing reduces the amount of inhibitory compounds (e.g. ABA) and improves germination performance (Michael Ignatz, UK). Seeds with high germination capacity likely have a high metabolic rate (MR), which can be determined using a modified Li-Cor system to determine $\mathrm{CO}_{2}$ production. As MR increases predictably with increasing viability of a seed lot, $\mathrm{O}_{2}$ consumption (measured using a Q2 scanner) decreases with increasing seed age (Emma Dalziell, Australia).

But not all facilities have access to advanced technologies to enable seed quality assessment or the maintenance of seed quality through the control of seed moisture. Local storage facilities for medium-term storage only, including for breeding materials, may lack drying rooms and refrigeration units, increasing the risk of post-harvest losses of grains at high $\mathrm{RH}$ from fungal and insect infestations and mycotoxin accumulation. In these situations, the implementation of the dry chain is recommended, involving drying to low eRH (e.g. using Drying Beads ${ }^{\mathrm{TM}}$ ) and waterproof packaging to maintain dryness during storage. The system is being implemented in seed and germplasm preservation programmes in India, Bangladesh and Mexico (Kent J. Bradford, USA). A spreadsheet gives the amount of Drying Beads required to dry a quantity (initial weight) of seeds based only on the initial seed $\mathrm{eRH}$ and the desired final $\mathrm{eRH}$ at the storage temperature, taking into account the oil content-dependent water-holding properties of seeds between 20 and $70 \% \mathrm{RH}$; and is available for lettuce, cucumber, pepper, tomato and onion seeds (Pedro Bello, USA). A different innovation concerns the use of two-step drying $\left(40^{\circ} \mathrm{C}\right.$ and $35 \% \mathrm{RH}$ first, then $17^{\circ} \mathrm{C}$ and $\left.15 \% \mathrm{RH}\right)$ on seed longevity of cowpea (Vigna unguiculata; Leguminosae). Both improvement to and negative effects of this drying on subsequent longevity are possible, partly dependent on seed harvest date (Olorunnisola F. Owoborode, Nigeria).

The search for innovations in the management of accessions is an ongoing process. Not surprisingly, there is great attraction in using knowledge about the ageing process to improve recovery after ex situ storage. Seeds stored for up to 10 years can be invigorated by $6 \mathrm{~h}$ of priming with $\mathrm{KNO}_{3}$ (maize) and $\mathrm{NaCl}$ (common bean), with the effect more evident in younger seeds, and a correlation between seed germination potential and peroxidase activity (Mariam Betsiashvili, Georgia). Similarly, after $8 \mathrm{~d}$ of high temperature and high humidity ageing, seeds of oats and barley primed at various concentrations of a catalytic seed treatment (composed of transition metals and $\mathrm{H}_{2} \mathrm{O}_{2}$ [CATTM]) have up to $30 \%$ more germination compared with unprimed ones (Solomon Sakyi-Quartey, Canada).

\section{An outlook on diversity}

While this perspective on seed longevity science represents a snapshot in time, it does highlight many interesting avenues of research that seem worthy of exploration in the future, inter alia:

(1) Opportunities for broad-scale comparative studies on seed longevity will increase as repeat testing of accessions held for decades in national and international seed collections extends to a wider and wider diversity. Beyond such longitudinal research, the detailed studies summarized here show that the range of biodiversity under investigation is still quite narrow. Approximately 60 species from 25 families studied represent about only $5.5 \%$ of all plant families. Interestingly, there is a keen interest in seed longevity as a means to an end, particularly the use of seeds in the restoration of degraded lands. With plant conservation and crop breeding, this is certainly another excellent way to describe a pathway to impact for seed longevity research.

(2) Many advances in understanding seed longevity have developed through investigations on three model and crop species: Arabidopsis thaliana, Oryza sativa and Medicago truncatula. The availability of mutants, the identification of QTLs and detailed signalling pathway studies have revealed some shared genetic bases controlling seed storability and dormancy, and a general role for oxidative stress in ageing. These two dicot and one monocot (rice) species have relatively small genomes in common, with 1C DNA amounts from around $0.3 \mathrm{pg}$ for Arabidopsis to around $0.5 \mathrm{pg}$ for the other two species (Leitch et al., 2019). Nowadays, C-values are available for 12,273 plant species, with a staggering genome size diversity that ranges around 2400-fold. This provides a framework for studies on genome evolution and seed longevity that should be explored.

(3) The huge variation in seed longevity reflects an interaction between the environmental signal during the later stages of seed development and genetics, particularly for rapid ageing conditions. Pursuing studies on model systems for which there is a reference genome advances understanding of which gene transcripts are particularly important to ageing and longevity, notwithstanding the potential for non-specific fragmentation in longer transcripts during ageing. Excitingly, 
$>200$ plant genomes have now been sequenced, including 181 horticultural species from $>60$ families and 30 orders of the plant kingdom (Chen et al., 2019). Assuming good homology between wild species and these reference genomes, and a greater understanding of trait variability through studying pan-genomes (available for Helianthus annuus, Capsicum spp., Solanum lycoperiscum, Glycine soja and Brassica oleracea), the unravelling of the genomics of seed longevity seems likely to progress rapidly.

(4) Preparing seeds for entry into the dry state is a complex integration of environmental signals that impact the timing of the acquisition of the longevity trait and affect biochemical pathways for the final chemical composition of the seed (under the control of the species' genetics). Thereafter, the storage environment imposes a physical architecture that is subject to relaxation and collapse, depending on the chemical reactivity and thermal properties of constituent parts and potentially in different parts of the cells. The unpredictability of such physical changes and associated alteration in molecular mobility seems to contribute to the heterogeneity of responses in dry, cold storage. But these changes are not easy to detect in space and time and much may be learned from the use of advanced biophysical and imaging techniques. The situation in the soil seed bank is somewhat different due to the opportunities in some habitats for the seeds to cycle through drier and wetter states, meaning periods of accumulated injury and activated repair. Considering species' biogeography and macroecology might then prove to be interesting in developing a more holistic, predictive framework for seed longevity.
Acknowledgement. The author thanks Henk Hilhorst, Christina Walters, Louise Colville, Anne Visscher and Dani Ballesteros for their helpful comments.

\section{References}

Chen F, Song Y, Li X, Chen J, Mo L, Zhang X, Lin Z and Zhang L (2019) Genome sequences of horticultural plants: past, present, and future Horticulture Research 6, 112. https://doi.org/10.1038/s41438-019-0195-6.

Colville L and Pritchard HW (2019) Seed life span and food security. New Phytologist 224, 557-562. https://doi.org/10.1111/nph.16006.

Hay FR, Valdez R, Lee J-S and Cruz PCS (2019) Seed longevity phenotyping: recommendations on research methodology. Journal of Experimental Botany 70, 425-434. https://doi.org/10.1093/jxb/ery358.

Leitch IJ, Johnston E, Pellicer J, Hidalgo O and Bennett MD (2019) Plant DNA C-values database (release 7.1). Available at: https://cvalues.science.kew.org/.

Liu U, Cossu TA, Davies RM, Forest F, Dickie JB and Breman E (2020) Conserving orthodox seeds of globally threatened plants ex situ in the Millennium Seed Bank, Royal Botanic Gardens, Kew, UK: the status of seed collections. Biodiversity and Conservation. https://doi.org/10.1007/ s10531-020-02005-6.

Mace GM, Barrett M, Burgess ND, et al. (2018) Aiming higher to bend the curve of biodiversity loss. Nature Sustainability 1, 448-451. https://doi.org/ 10.1038/s41893-018-0130-0.

Solberg S $\emptyset$, Yndgaard F, Andreasen C, von Bothmer R, Loskutov IG and Asdal Å (2020) Long-term storage and longevity of orthodox seeds: a systematic review. Frontiers in Plant Science 11, 1007. https://doi.org/10.3389/ fpls.2020.01007.

Zinsmeister J, Leprince O and Buitink J (2020) Molecular and environmental factors regulating seed longevity. Biochemical Journal 477, 305-323. https:/ doi.org/10.1042/BCJ20190165. 